\title{
A Novel Approach to Evaluate the Extent and the Effect of Cross-Contribution to the Intensity of Ions Designating the Analyte and the Internal Standard in Quantitative GC-MS Analysis
}

\author{
Bud-Gen Chen, ${ }^{\mathrm{a}}$ Chiung Dan Chang, ${ }^{\mathrm{b}}$ Chia-Ting Wang, ${ }^{\mathrm{c}}$ Yi-Jun Chen, ${ }^{\mathrm{d}}$

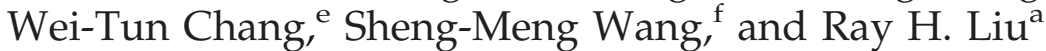 \\ a Department of Medical Technology, Fooyin University, Kaohsiung, Taiwan \\ ${ }^{\mathrm{b}}$ Department of Laboratory, Yang Ming Hospital, Chiayi, Taiwan \\ c Department of Laboratory, Ben Tang Cheng Ching Hospital, Taichung, Taiwan \\ d Department of Pathology, Chang Bing Show Chwan Memorial Hospital, Changhua, Taiwan \\ ${ }^{\mathrm{e}}$ Department of Criminal Investigation, Central Police University, Taoyuan, Taiwan \\ ${ }^{\mathrm{f}}$ Department of Forensic Sciences, Central Police University, Taoyuan, Taiwan
}

In gas chromatography-mass spectrometry methods of analysis adopting the analyte's isotopic analog as the internal standard (IS), the cross-contribution (CC) phenomenon-contribution of IS to the intensities of the ions designating the analyte, and vice versa- has been demonstrated to affect the quantitation data. A novel approach based on the deviations of the empirically observed concentrations of a set of standards was developed to assess the accuracy of the empirically derived CC data. This approach demonstrated that normalization of ion intensities derived from the analyte and the IS generates reliable CC data. It further demonstrated that an ion-pair (designating the analyte and the IS) with $\sim 5 \%$ or higher CC will result in a very limited linear calibration range. (J Am Soc Mass Spectrom 2008, 19, 598-608) (c) 2008 American Society for Mass Spectrometry

I n 1984, guidelines were established for the U.S. Federal Workplace Drug Testing Program, mandating (1) specific "cutoff" concentrations as positive/ negative criteria, and (2) certain concentration-related quality control and method validation requirements [1]. Accurate quantitation of drugs/metabolites in biological specimens has since, in addition to being a scientific pursuit, evolved into a legal issue.

Selected ion monitoring (SIM) has long been established as the most effective approach for data collection where gas chromatography-mass spectrometry (GCMS) is used for the quantitation of various categories of analytes. Among various calibration approaches applied to SIM GC-MS protocols, internal standard (IS) method using isotopically-labeled analog (ILA) of the analyte as the IS has been well studied [2-7] and now widely adopted in forensic, clinical, and environmental laboratories. With ILA as the IS, one area of concern is the ion intensity cross-contribution (CC) between the analyte and the IS.

Cross-contribution is defined as the contribution of the IS to the intensities of the ions designating the

Address reprint requests to Professor Ray H. Liu, Department of Medical Technology, Fooyin University, 151 Ching-Hsueh Road, Ta-Liao Hsiang, Kaohsiung Hsien 831-02, Taiwan. E-mail: mt124@mail.fy.edu.tw analyte and vice versa. Since the measured ion intensities are used for the quantitation of the analyte, adopting an ion-pair with significant CC to designate the analyte and the IS will generate inaccurate analyte concentrations. For example, when the contribution of the IS to the intensity of the ion designating the analyte is more significant, the observed apparent analyte concentration will be higher than its true value. This error will become more significant as the analyte's concentration is lowered. On the other hand, the observed apparent analyte concentration will be lower than its true value when the analyte's contribution to the intensity of the ion designating the IS is more significant. Similarly, this error will become more significant as the analyte's concentration is increased.

Theoretical considerations [4] and approaches involving high-resolution ion monitoring [5] and computer programming for deconvoluting mass spectral peak abundance $[6,7]$ have been reported. The need to address this phenomenon in "real world data" was also highlighted by the inclusion of a section entitled, "Corrections for Contamination and Isotope Spillover," in a 2006 book by Duncan et al. [8]. In their book, the authors illustrated a nonlinear relationship (Figure 8.4) between the monitored response and the analyte concentration, and further demonstrated (Figure 8.3) that a 
linear relationship can be expected by removing the portion of the intensity of the ions designating the analyte that was cross-contributed by (spillover from) the IS (and vice verse).

Our interest in this area includes empirical measurements of the CC data [9-11], characterization of the effect of CC on the calibration curve [12], and the generation of favorable ion-pairs for designating the analyte and the IS, mainly through various chemical derivatization (CD) routes [13].

The CC phenomenon has long been recognized and, as mentioned above, many correction approaches have been reported. However, to the best of our knowledge, assessing the accuracy (trueness) of the empirically determined CC data, which could have been affected by systematic and random errors, has not been addressed. This study develops a novel approach to evaluate empirically-derived CC values, advancing current knowledge in this important analytical parameter.

\section{Experimental}

\section{Standards and Reagents}

The following analytes and deuterated ISs (in 1 or 0.1 $\mathrm{mg} / \mathrm{mL}$ methanol solution) were purchased from Cerilliant Corp., Austin, TX: 3,4-methylenedioxyamphetamine (MDA), hydromorphone (HM), MDA- $\mathrm{d}_{5}$, and hydromorphone- $\mathrm{d}_{6}\left(\mathrm{HM}-\mathrm{d}_{6}\right)$. Derivatization reagents, $N$-methyl- $N$-( $t$-butyldimethylsilyl)trifluoroacetamide (with 1\% t-butyldimethyl-chlorosilane) and $\mathrm{N}, \mathrm{O}$-bis(trimethylsilyl)trifluoroacetamide with (1\% ttrimethylchlorosilane), were purchased from Pierce Chemical Co., Rockford, IL. All other common chemicals and solvents were of HPLC grade.

\section{Sample Preparation and Derivatization Procedure}

For full-scan and SIM data collection, the analytes (MDA and HM) and the ISs (MDA- $\mathrm{d}_{5}$ and $\mathrm{HM}-\mathrm{d}_{6}$ ) solutions were prepared individually. For example, for the run including only MDA, $5 \mu \mathrm{L}$ of the MDA standard $(1 \mathrm{mg} / \mathrm{mL}$ in methanol) was transferred into a $16 \times$ $100-\mathrm{mm}$ glass tube. For the run including only MDA- $\mathrm{d}_{5}$, $50 \mu \mathrm{L}$ of the MDA- $\mathrm{d}_{5}$ standard $(0.1 \mathrm{mg} / \mathrm{mL}$ methanol solution) was used. Thus, an equal amount of the MDA and MDA- $\mathrm{d}_{5}$ was used in these two parallel experiments.

The procedures described below were then followed to form the $t$-butyldimethylsilyl ( $t$-BDMS) or the trimethylsilyl (TMS) derivatives of the analytes and the ISs. The $16 \times 100$-mm glass tube containing the analyte or the IS as prepared in the last paragraph was evaporated to dryness under a stream of nitrogen at $50{ }^{\circ} \mathrm{C}$. To the dried residue was added $50 \mu \mathrm{L}$ acetonitrile and 50 $\mu \mathrm{L}$ of the selected derivatization reagent; the tube was capped, mixed, and incubated for $20 \mathrm{~min}$ at $90{ }^{\circ} \mathrm{C}$ in a heating block [9]. The mixture was cooled for GC-MS analysis. The structures of the derivatized analytes and ISs are shown in Figure 1 along with their mass spectra.

\section{Instrumentation, Analytical Parameters, and Data Collection Procedure}

GC-MS analysis was performed on an Agilent 6890 GC interfaced to an Agilent 5975 MSD (Agilent, Palo Alto, CA). A 12-m HP-ULTRA-1 crosslinked 100\% methyl siloxane capillary column $(0.20-\mathrm{mm}$ i.d., $0.33-\mu \mathrm{m}$ film thickness) from Agilent (Wilmington, DE) was used for this study. Helium carrier gas flow rate was $1.0 \mathrm{~mL} /$ min. The injector and GC-MS interface temperatures were maintained at $250{ }^{\circ} \mathrm{C}$ and $280{ }^{\circ} \mathrm{C}$, respectively. For MDA experiment, the GC oven temperature was initiated at $75{ }^{\circ} \mathrm{C}$ (held for $0.5 \mathrm{~min}$ ), raised to $200^{\circ} \mathrm{C}$ at $20^{\circ} \mathrm{C} / \mathrm{min}$ (held for $1 \mathrm{~min}$ ), then to $275^{\circ} \mathrm{C}$ at $40^{\circ} \mathrm{C} / \mathrm{min}$ (held for $1 \mathrm{~min}$ ); for $\mathrm{HM}$, the GC oven temperature was initiated at $160{ }^{\circ} \mathrm{C}$ (held for $1 \mathrm{~min}$ ), raised to $250{ }^{\circ} \mathrm{C}$ at $20^{\circ} \mathrm{C} / \mathrm{min}$ (held for $3 \mathrm{~min}$ ), then to $290^{\circ} \mathrm{C}$ at $10^{\circ} \mathrm{C} / \mathrm{min}$ (held for $2 \mathrm{~min}$ ). These oven temperature programming parameters are obviously more than what are needed for this study; however, they are routinely used in this laboratory for the analyses of respective categories of compounds and have been adopted here to facilitate the identification of specific derivatization products.

Typically, a full-scan mass spectrum of the derivatized analyte or IS was obtained by injecting the CD product into the GC-MS system. The scan-range was typically set from $\mathrm{m} / \mathrm{z} 50$ to the molecular weight of the anticipated product with the maximal number of $C D$ groups, rounded to the next "50" or "100." A separate run was repeated for the isotopic analog of the analyte. Information derived from these ion chromatograms (retention time and mass spectrometric data) were used to characterize the analyte or the IS. Full-scan mass spectrometric data were stored as digital files that were then converted into mass spectra of a more desirable format for systematic presentation as shown in Figure 1. This conversion was carried out using DeltaGraph software (DeltaPoint, Seattle, WA) on an Apple iMac G5 computer (Cupertino, CA).

Full-scan mass spectrometric data obtained from these runs were reviewed to select ions (Figures 2 and 3) that may be suitable for designating the analyte and its IS in routine GC-MS protocols. These CD products (the analyte of interest and its isotopic analogs) were injected (separately) into the GC-MS again under SIM mode, and the ions selected from the full-scan mass spectrometric data were monitored. General criteria adopted for SIM ion selection included: (1) full-scan intensity data indicated less than 10\% CC; and (2) the ion's relative intensity in the full-scan mass spectrum was $\geq 10 \%$. Ions with lower intensity would have been included if there were less than three pairs of ions that met the above criteria. Mass spectrometric data derived from these SIM runs were then used to evaluate the CC data. Details of the methodology have been described in 

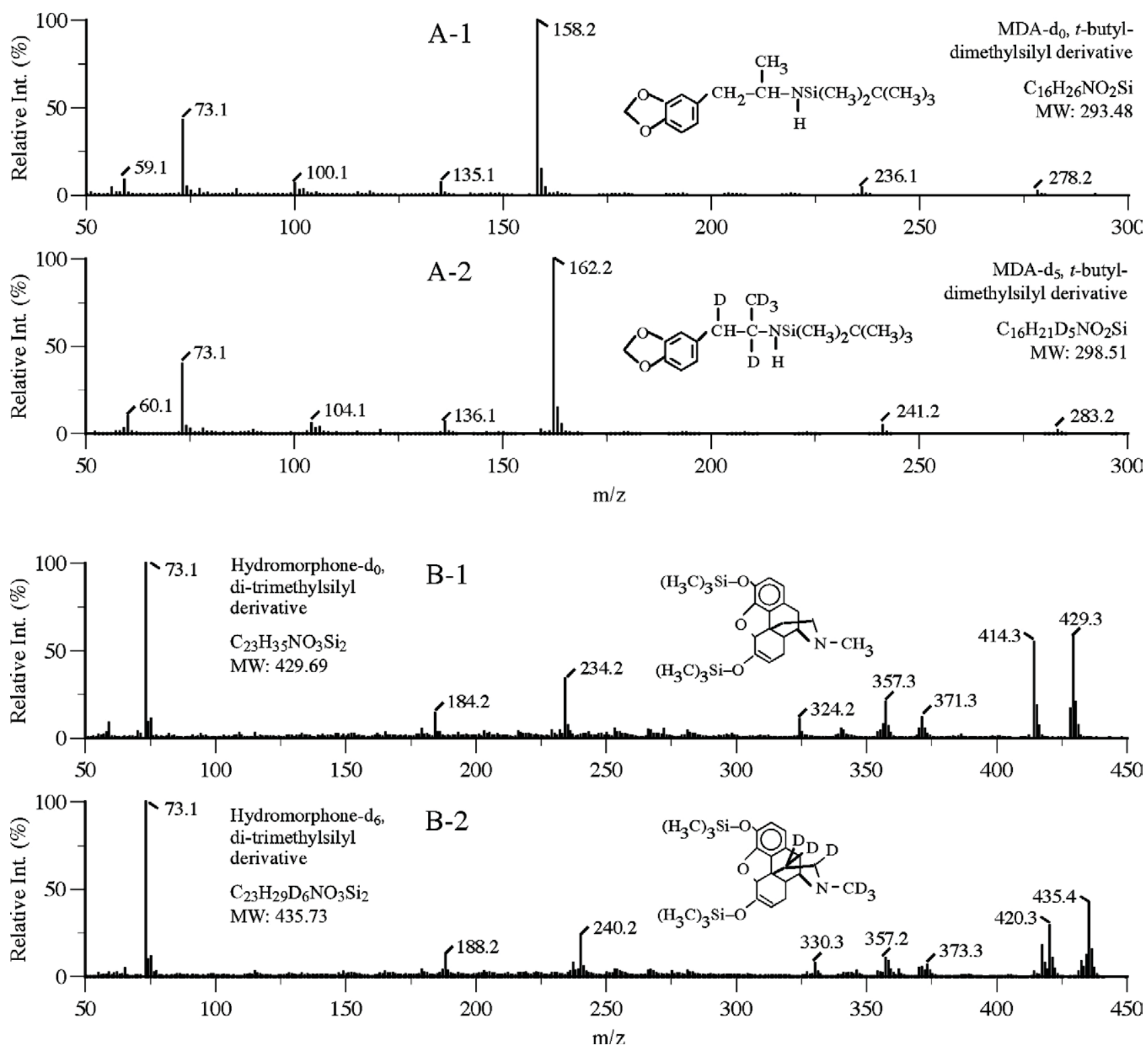

Figure 1. Full-scan mass spectra and molecular information of (A) MDA/MDA- $\mathrm{d}_{5}$ (as $t$-BDMS derivatives) and (B) hydromorphone/hydromorphone- $\mathrm{d}_{6}$ (as TMS derivatives). (All spectra were obtained using individual components.)

our earlier publications $[9,10]$ and briefly illustrated in the next section using the data derived from the MDA/ MDA- $\mathrm{d}_{5}$ system as the example.

\section{Normalization of SIM Data Derived from the Analyte and the IS and the Calculation of CC Data}

Full-scan mass spectra of $t$-BDMS derivatized MDA and MDA- $d_{5}$ (Figure 1) indicate the following ion-pairs meet the selection criteria described in the last paragraph: $m / z$ 100/104, 158/162, 236/241, 278/283 (Figure 2 ). They were further examined by the SIM protocol. Shown in Table 1 is one set of the observed raw ion intensity data (in \% relative intensity for full-scan; in integrated peak area for SIM). Also included in this table are the normalized SIM data (for MDA- $\mathrm{d}_{5}$ ) and the CC data calculated based on the raw and the normalized data. Example calculations for the normalization process and the derivation of CC data are shown below.

For the SIM run including only MDA, the intensity of the base-peak ion for MDA ( $m / z$ 158) was 20,146,666. On the other hand, the intensity for the corresponding ion $\left(\mathrm{m} / z\right.$ 162) for MDA- $\mathrm{d}_{5}$, in the run including only MDA$\mathrm{d}_{5}$, was $40,018,206$. Thus, all SIM ion intensity data derived from the run including only $\mathrm{MDA}^{-\mathrm{d}_{5}}$ were adjusted by a factor of 20,147,110/40,018,206 (or 0.5034) and shown in the last column in Table 1. For example, the normalized intensity for the ion $\mathrm{m} / \mathrm{z} 100$ collected 


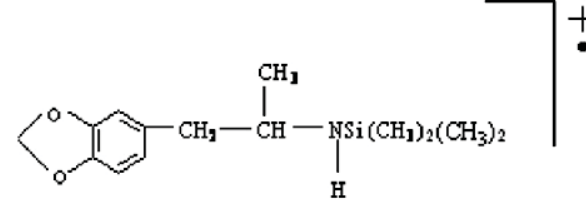

$\mathrm{M}-15=278$ (or 283 for IS)

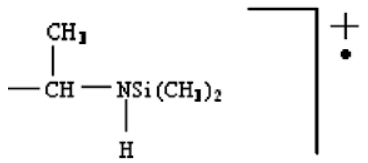

M-193/M-194 = 100

(or 104 for IS)

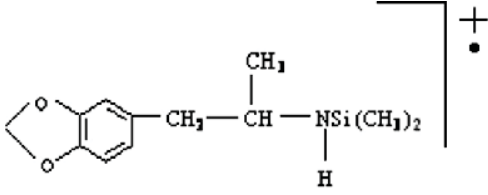

$\mathrm{M}-57=236$ (or 241 for IS)

Figure 2. Fragments of major ions derived from MDA (as $t$-BDMS derivatives).

during the run including only MDA- $\mathrm{d}_{5}$ is now $76,615 \times$ 0.5034 (or 38,568).

Two sets of CC data were then calculated, both using the raw data generated from the run including only MDA, but using the raw or the normalized data generated from the run including only MDA- $\mathrm{d}_{5}$. For example, for the ion-pair $m / z$ 100/104, the run including only MDA generated an intensity value of 1291,998 , while the run including only MDA- $\mathrm{d}_{5}$ generated an intensity value of 76,615 ; thus, MDA- $\mathrm{d}_{5}$ would have contributed 76,615/1291,998 = $5.93 \%$ to the intensity measured for $m / z 100$, if an equal amount of MDA and MDA- $\mathrm{d}_{5}$ were present. (It should be pointed out that the CC hereby defined differs from what has been adopted by Barbalas and Garland [7]. Specifically, we define the CC of the IS to the analyte as the intensity ratio of this ion generated by the IS to that generated by the analyte, when equal amounts of the IS and the analyte are present. For their purpose, Barbalas and Garland defined a "coefficient", which is the intensity ratio of the ion designating the analyte to that designating the IS, when only the IS is present.)

The second set of CC data were calculated using the normalized ion intensity for MDA- $\mathrm{d}_{5}$. Since the normalized intensity for the ion $\mathrm{m} / \mathrm{z} 100$ collected during the run including only MDA- $\mathrm{d}_{5}$ is 38,568 , the percent CC calculated based on the normalized data is $38,568 /$ $1291,998=2.99 \%$. Both sets of CC data $(5.93 \%$ and $2.99 \%$ ) are shown in Table 1.

\section{Results and Discussion}

In a 1989 study [14] on the quantitation of benzoylecgonine (a cocaine metabolite), it was noted that the CC phenomenon between the ion-pair (ions designating the analyte and the IS) systematically affected the resulting quantification data. Accordingly, a procedure was developed in a later study [9] to determinate the CC of ion-pairs that may potentially be used to designate the analyte and the selected IS. With these data available, it was then possible to select the ion-pair with no (or minimal) CC for the quantitation purpose. However, whether the method developed indeed produced accurate CC values has always been an area of concern. Thus, a follow-up study [10] was conducted to develop three additional methods (improved direct measurement, internal standard, and standard addition), and they were compared against the method (direct measurement) developed earlier [9]. This later study [10] concluded that "all methods produce practically the same order, among ions derived from each isotopic analog, in their extents in contributing to the intensities of respective ions designated for a specific counter isotopic analog. Thus, all methods can be used to select the best ion-pair within a selected analyte/[IS] for the intended quantitative analysis protocol" [10]. However, the accuracy (trueness) of the empirically determined CC data still could not be assessed. With this in mind, the approach described below is proposed to deter-

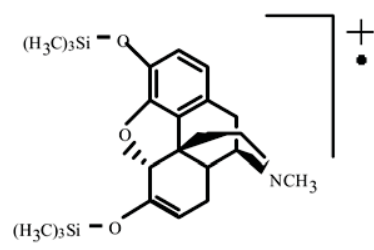

$M=429$ (or 435 for IS)

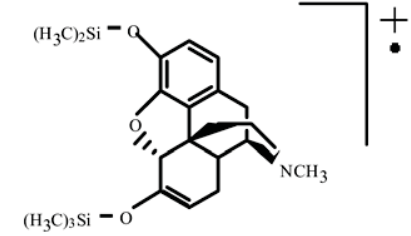

M-15 = 414 (or 420 for IS)

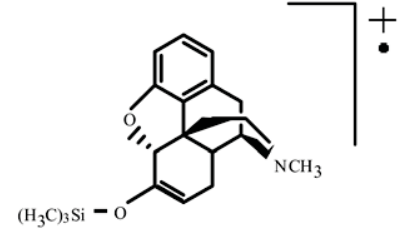

$M-105=324$ (or 330 for IS)

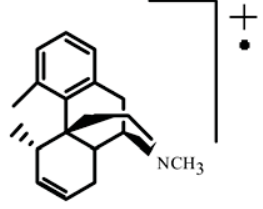

$\mathrm{M}-195=234$ (or 240 for IS)

Figure 3. Fragments of major ions derived from HM (as TMS derivatives). 
Table 1. MDA and MDA- $\mathrm{d}_{5}$ ion intensity data collected under full-scan (in \%) and SIM (peak area) mode ${ }^{\mathrm{a}}$

\begin{tabular}{|c|c|c|c|c|c|}
\hline \multirow[b]{2}{*}{$\begin{array}{l}\text { Ion } \\
(\mathrm{m} / \mathrm{z})\end{array}$} & \multicolumn{2}{|c|}{ Raw data from the MDA run } & \multicolumn{2}{|c|}{ Raw data from the MDA- $d_{5}$ run } & \multirow{2}{*}{$\begin{array}{c}\text { Normalized data for MDA- } d_{5} \\
\text { SIM ion intensity } \\
\text { (\% CC by MDA) }\end{array}$} \\
\hline & $\begin{array}{l}\text { Full-scan } \\
\text { (rel. int.) } \\
\end{array}$ & $\begin{array}{l}\text { SIM ion intensity } \\
\left(\% \text { CC by MDA- } d_{5}\right)\end{array}$ & $\begin{array}{l}\text { Full-scan } \\
\text { (rel. int.) }\end{array}$ & $\begin{array}{l}\text { SIM ion intensity } \\
\text { (\% CC by MDA) }\end{array}$ & \\
\hline \multicolumn{6}{|c|}{ lons designating MDA } \\
\hline 100 & $6.6 \%$ & $1,291,998(5.93 \% ; 2.99 \%)^{\mathrm{b}}$ & $0.4 \%$ & 76,615 & 38,568 \\
\hline 158 & $100 \%$ & $20,146,666(0.10 \% ; 0.05 \%)$ & $0.1 \%$ & 19,859 & 9,997 \\
\hline 236 & $4.0 \%$ & $857,201(1.024 \% ; 0.52 \%)$ & $0.0 \%$ & 8,777 & 4,418 \\
\hline 278 & $2.1 \%$ & $472,570(0.25 \% ; 0.13 \%)$ & $0.0 \%$ & 1,179 & 594 \\
\hline \multicolumn{6}{|c|}{ lons designating MDA- $d_{5}$} \\
\hline 104 & $0.2 \%$ & 88,342 & $5.8 \%$ & $2,247,498(3.93 \%)$ & $1,131,390(7.81 \%)$ \\
\hline 162 & $0.3 \%$ & 109,115 & $100 \%$ & $40,018,206(0.27 \%)$ & $20,147,165(0.54 \%)$ \\
\hline 241 & $0.0 \%$ & 158 & $4.4 \%$ & $1,938,632(0.01 \%)$ & $975,907(0.02 \%)$ \\
\hline 283 & $0.0 \%$ & 248 & $2.0 \%$ & $913,091(0.03 \%)$ & $459,650(0.05 \%)$ \\
\hline
\end{tabular}

all data shown in this table were obtained from a single experiment. Reproducibility data are shown in Table 2.

bData shown inside parentheses are CC data (in \%). When two CC data are given, the first and the second data were derived, respectively, from raw and normalized ion intensity data.

mine whether a set of empirically determined $\mathrm{CC}$ is indeed accurate.

\section{Assessing the Accuracy of the Empirically Determined Cross-Contribution Values}

A four-step process was developed to assess whether a set of empirically derived CC data for a specific ion-pair designating an analyte/IS system are correct. Steps of this approach are first outlined below, while details of each step have either been described in the Experimental section or will be further illustrated later: (1) the CC of an selected ion-pair were determined using the raw ion intensity data [9], followed by the calculation of another set of CC data using normalized ion intensity data; (2) a series of standard solutions were prepared and then analyzed to obtain a set of experimentally observed concentrations; (3) the two sets of CC data (derived from raw and normalized ion intensity data) were alternately used to derive two sets of theoretically calculated concentrations for this set of standards; and finally, (4) these three sets of concentrations were evaluated to determine if either set of the theoretically calculated concentrations is the same (allowing experimental errors) as the set of the experimentally observed concentrations.

The concentrations of individual standards in each set deviated from their respective true values, but with different implications. Deviations of the experimentally observed concentrations were caused by the true CC imbedded in the adopted ion-pair designating the analyte and the IS, while deviations, if any, of the theoretically calculated concentrations were caused by incorporating incorrect empirically determined CC values (two sets) into the calculation. Thus, if the set of CC data under examination are accurate, these deviations resulting from the theoretically calculated data, using this set of CC, should coincide well with the experimentally observed (permitting random experimental errors). On the other hand, significant differences between these two sets of deviations indicate existence of significant random and/or systematic errors in deriving this set of CC data under examination.

For this study, the MDA/MDA- $\mathrm{d}_{5}$ system was selected as the exemple analyte/IS system and $t$-BDMS the derivatization group. Data derived from this system will be fully presented to illustrate the details of the approach, while only the concluding data for the HM/ $\mathrm{HM}-\mathrm{d}_{6}$ system (with TMS as the derivatization group) will be presented to support the validity of the approach hereby reported.

\section{Ion-Pair Selection and Ion Intensity Measurement}

Shown in Figure 2 are the fragments of major ions observed in the mass spectrum derived from MDA. The first four ions retain the structural framework in where most (or all) labeling deuterium atoms are positioned; they can potentially be used to designate the analyte and the IS. The last ion, $[\mathrm{M}-158]^{+}$(or $m / z$ 135) for MDA and $[\mathrm{M}-162]^{+}$(or $m / z$ 136) for MDA- $\mathrm{d}_{5}$, differ only by one atomic unit and, thus, is not suitable for designating the analyte and the IS. Ion $m / z 73$ comes from the derivatization group (TMS, $t$-BDMS). It is not characteristic of the analyte of interest and cannot be used to designate the analyte and the IS.

Cross contribution data shown in Table 1 indicate that the most favorable ion-pair for designating MDA/ MDA- $\mathrm{d}_{5}$ is $m / z 158 / 162$. Both ions in this pair exhibit high intensities and low CC; thus, they were selected as the control in this study to illustrate the generation of high-quality quantitation data. Ions $m / z$ 100/104 have reasonable intensities, but also with significant CC. Adopting this ion-pair for quantitation will result in noticeable error. Since the main objective of this study is to examine the interference consequence of $\mathrm{CC}$ in the quantitation process, ion-pair $m / z$ 100/104 (with significant (C) was selected to fully illustrate the deviation phenomenon and the assessment process as outlined in the first paragraph of the last subsection. The other two 
Table 2. Precision of cross contribution data derived from within- and between-day measurements

\begin{tabular}{|c|c|c|c|c|c|c|c|c|c|c|}
\hline \multirow[b]{3}{*}{ Ion } & \multicolumn{4}{|c|}{ Day 1} & \multicolumn{4}{|c|}{ Day 3} & \multicolumn{2}{|c|}{ Days 1 and 3} \\
\hline & \multicolumn{3}{|c|}{ Raw data } & \multirow{2}{*}{$\frac{\text { Normalized }}{\text { Mean }}$} & \multicolumn{3}{|c|}{ Raw data } & \multirow{2}{*}{$\frac{\text { Normalized }}{\text { Mean }}$} & \multirow{2}{*}{$\frac{\text { Raw data }}{\text { Mean }}$} & \multirow{2}{*}{$\frac{\text { Normalized }}{\text { Mean }}$} \\
\hline & Mean $^{a}$ & SD & $\mathrm{CV} \%$ & & Mean $^{a}$ & SD & $\mathrm{CV} \%$ & & & \\
\hline \multicolumn{11}{|c|}{ lons designating MDA } \\
\hline 100 & 5.85 & 0.82 & 14 & 3.02 & 5.98 & 1.40 & 23 & 2.96 & 5.92 & 2.99 \\
\hline 158 & 0.11 & 0.02 & 19 & 0.06 & 0.17 & 0.06 & 36 & 0.08 & 0.14 & 0.07 \\
\hline \multicolumn{11}{|c|}{ lons designating MDA- $\mathrm{d}_{5}$} \\
\hline 104 & 4.22 & 0.46 & 11 & 8.09 & 4.16 & 0.87 & 21 & 8.08 & 4.19 & 8.09 \\
\hline 162 & 0.27 & 0.04 & 15 & 0.51 & 0.30 & 0.08 & 27 & 0.59 & 0.28 & 0.55 \\
\hline
\end{tabular}

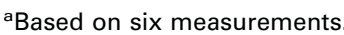

ion-pairs, $m / z$ 236/241 and 278/283, will not be discussed further.

Ion intensities derived from MDA and MDA- $\mathrm{d}_{5}$ may not be compatible for the following reasons: (1) due to experimental errors, the quantities of MDA and MDA- $\mathrm{d}_{5}$ may not be exactly the same; (2) the derivatization reaction for MDA and MDA- $\mathrm{d}_{5}$, performed in separate tubes, may not be completed to the same extent; and (3) the separate injections and data collections for MDA and MDA- $\mathrm{d}_{5}$ in the GC-MS process may also involve variations. For these reasons, the $\mathrm{CC}$ data calculated based on the raw intensity data produced in two separate runs for MDA and MDA- $\mathrm{d}_{5}$ may include bias. Thus, alternatively, intensity data of MDA- $\mathrm{d}_{5}$ ions were converted into "normalized" values with the assumption that the base-peak ions $(\mathrm{m} / \mathrm{z} 158$ and 162 in this case) of the MDA and MDA- $\mathrm{d}_{5}$ would have the same intensities (the normalized intensity data are shown in the last column in Table 1).

The normalization process was intended to correct experimental errors. Systematic factors, such as isotopic effect on the ion fragmentation process that might have been caused by the deuterium atoms in MDA- $\mathrm{d}_{5}$, have not been addressed. If the isotopic effect is a significant factor, the CC of MDA toward the intensities of ions designating MDA- $_{5}$ (and vice versa) would have been under- or over-estimated depending on whether the isotope effect would reduce or enhance the intensities of the corresponding ion fragments derived from MDA$d_{5}$. Thus, assessing the CC data derived from the normalization process can also reveal whether isotope effect is a significant factor in the system under examination.

\section{Multiple Measurements for Precision Study}

The reproducibility of the calculated CC data were examined at two levels. First, each of the run including only MDA and the run including only $\mathrm{MDA}^{-\mathrm{d}_{5}}$ was injected into the GC-MS system six times. The resulting ion intensity data were used for the calculation of the $\mathrm{CC}$ data as described in the Experimental section. At the second level, a new set of MDA and $M D A-d_{5}$ was prepared individually in a different day and again each injected six times. Mean, standard deviation, and coefficient of variation ( $\mathrm{CV} \%$ ) for the $\mathrm{CC}$ data derived from the raw ion intensity data are shown in Table 2. The corresponding means of CC data derived from the normalized ion intensity data are also included in the table. Corresponding data derived from days 1 and 3 were averaged and entered into the last two columns of the table. All CC data shown in Tables 1 and 2 were rounded to the second digit after the decimal point. Cross contribution at this level (one hundredth of $1 \%$ ) will not contribute to observable difference in practical applications.

The precisions of the resulting CC data were assessed by the observed standard deviation and $\mathrm{CV} \%$ of these measurements. As shown in Table 2, the CV\% for $\mathrm{CC}$ in the parts per hundred range were between $11 \%$ and $23 \%$, while the $\mathrm{CV} \%$ for the CC data in the parts per thousand range were between $15 \%$ and $36 \%$. These precision data are not poor ones, considering the fact that each CC value was derived from a highly abundant ion in one run and a very low abundant ion in another run. This is especially true for the $C C$ in the parts per thousand range and, in this case, it should not be a matter of concern for the following reasons: (1) the intensities of the CC ions are negligibly, making their precise measurement impossible; and, perhaps more importantly, (2) with such low CC, quantitation data resulting from the adoption of these ion-pairs would not really be affected. This latter statement is further supported by the exemplar data shown in the next section.

\section{Selection of Ion-Pairs and Calibration Method}

Shown in Table 3 are two sets of data (for ion-pairs $m / z$ 100/104 and 158/162) derived from a series of standard solutions prepared in drug-free urine with the concentrations of MDA ranging from 30 to $4000 \mathrm{ng} / \mathrm{mL}$. As mentioned earlier, ion-pair $m / z$ 158/162 exhibits minimal CC; thus, adopting this ion-pair for quantitation can generate high-quality data. Data are shown in the lower section of Table 3 to serve as a control, indicating deviations resulting from the ion-pair $m / z$ 100/104 are indeed caused by the significant CC imbedded in the 
Table 3. Effect of cross contribution on empirically determined and theoretically calculated concentrations of a series of standard solutions

\begin{tabular}{|c|c|c|c|c|c|c|}
\hline \multirow[b]{3}{*}{$\begin{array}{l}\text { Theoretical } \\
\text { conc. }\end{array}$} & \multirow{2}{*}{\multicolumn{2}{|c|}{ Empirically observed }} & \multicolumn{4}{|c|}{ Theoretically calculated with $\mathrm{CC}$ derived from } \\
\hline & & & \multicolumn{2}{|c|}{ Raw ion intensity data } & \multicolumn{2}{|c|}{ Normalized ion intensity data } \\
\hline & $\begin{array}{l}\text { Ion int. } \\
\text { ratio }\end{array}$ & $\begin{array}{l}\text { Observed conc. } \\
\text { (\% deviation) }\end{array}$ & $\begin{array}{l}\text { Ion int. } \\
\text { ratio }\end{array}$ & $\begin{array}{c}\text { Calculated conc. } \\
\text { (\% deviation) }\end{array}$ & $\begin{array}{l}\text { Ion int. } \\
\text { ratio }\end{array}$ & $\begin{array}{c}\text { Calculated conc. } \\
\text { (\% deviation) }\end{array}$ \\
\hline \multicolumn{7}{|l|}{$m / z 100 / 104^{a}$} \\
\hline 30 & 0.1425 & $42.8(42.5)$ & 0.0947 & $79.7(165.8)$ & 0.0978 & $42.9(42.9)$ \\
\hline 50 & 0.1944 & $58.3(16.6)$ & 0.1183 & $99.6(99.2)$ & 0.1427 & $62.5(25.1)$ \\
\hline 80 & 0.3085 & $92.5(15.7)$ & 0.1536 & $129.4(61.7)$ & 0.2094 & $91.7(14.7)$ \\
\hline 100 & 0.3978 & $119.3(19.3)$ & 0.1770 & $149.1(49.1)$ & 0.2534 & $111.1(11.1)$ \\
\hline 200 & 0.6713 & $201.4(0.68)$ & 0.2937 & $247.4(23.7)$ & 0.4689 & $205.5(2.8)$ \\
\hline 300 & 1.083 & $324(8.3)$ & 0.4093 & $344.7(14.9)$ & 0.6770 & $296.7(-1.1)$ \\
\hline 500 & $1.667^{\mathrm{b}}$ & 500 (Calibrator) & 0.6370 & $536.5(7.3)$ & 1.0719 & $469.7(-6.1)$ \\
\hline 800 & 2.525 & $757.3(-5.3)$ & 0.9703 & $817.3(2.2)$ & 1.617 & $708.5(-11.4)$ \\
\hline 1,000 & 3.149 & $944.5(-5.5)$ & 1.187 & $1,000(0.012)$ & 1.952 & $855.2(-14.5)$ \\
\hline 1,300 & 3.336 & $1,000(-23)$ & 1.5052 & $1,268(-2.5)$ & 2.416 & $1059(-18.5)$ \\
\hline 1,700 & 4.037 & $1,211(-28.8)$ & 1.915 & $1,613(-5.09)$ & 2.975 & $1304(-23.3)$ \\
\hline 2,000 & 4.510 & $1,353(-32.4)$ & 2.213 & $1,864(-6.8)$ & 3.355 & $1470(-26.5)$ \\
\hline 3,000 & 5.786 & $1.736(-42.1)$ & 3.151 & $2,654(-11.5)$ & 4.425 & $1939(-35.4)$ \\
\hline 4,000 & 6.726 & $2,017(-49.6)$ & 4.0101 & $3,378(-15.6)$ & 5.268 & $2309(-42.3)$ \\
\hline \multicolumn{7}{|l|}{$\mathrm{m} / \mathrm{z} 158 / 162^{\mathrm{c}}$} \\
\hline 30 & 0.0832 & $25.5(-15)$ & 0.0326 & $31.3(4.5)$ & 0.0607 & $30.3(1.1)$ \\
\hline 50 & 0.1370 & $42.0(-16)$ & 0.0534 & $51.3(2.7)$ & 0.1006 & $50.3(0.64)$ \\
\hline 80 & 0.2354 & $72.2(-9.8)$ & 0.0845 & $81.3(1.7)$ & 0.1606 & $80.3(0.35)$ \\
\hline 100 & 0.3107 & $95.3(-4.7)$ & 0.1053 & $101.3(1.3)$ & 0.2005 & $100.2(0.24)$ \\
\hline 200 & 0.5920 & $181.5(-9.3)$ & 0.2092 & $201.2(0.61)$ & 0.3998 & $199.9(-0.045)$ \\
\hline 300 & 0.9828 & $301.3(0.43)$ & 0.3129 & $301.1(0.36)$ & 0.5987 & $299.4(-0.21)$ \\
\hline 500 & $1.631^{\mathrm{b}}$ & 500 (Calibrator) & 0.5203 & $500.6(0.12)$ & 0.9952 & $497.6(-0.48)$ \\
\hline 800 & 2.723 & $834.8(4.3)$ & 0.8310 & $799.5(-0.064)$ & 1.587 & $793.4(-0.83)$ \\
\hline 1000 & 3.645 & $1,118(11.8)$ & 1.038 & $998.4(-0.16)$ & 1.979 & $989.5(-1.1)$ \\
\hline 1300 & 3.969 & $1,217(-6.4)$ & 1.348 & $1296(-0.27)$ & 2.564 & $1282(-1.4)$ \\
\hline 1700 & 5.196 & $1,593(-6.3)$ & 1.760 & $1693(-0.41)$ & 3.338 & $1669(-1.8)$ \\
\hline 2000 & 6.169 & $1,891(-5.4)$ & 2.068 & $1990(-0.51)$ & 3.915 & $1957(-2.1)$ \\
\hline 3000 & 9.593 & $2,941(-1.9)$ & 3.093 & $2975(-0.82)$ & 5.809 & $2905(-3.2)$ \\
\hline 4000 & 12.59 & $3,860(-3.5)$ & 4.111 & $3955(-1.1)$ & 7.664 & $3832(-4.2)$ \\
\hline
\end{tabular}

${ }^{a}$ These data were taken from the MDA/MDA- $d_{5}$ system using the raw ion intensity data to calculate the CC data: the contribution of MDA- $d_{5}$ to the intensities of ions designating MDA are $5.92 \%$ for $\mathrm{m} / \mathrm{z} 100$ and $0.14 \%$ for $\mathrm{m} / \mathrm{z} 158$; the contribution of MDA to the intensities of ions designating MDA- $\mathrm{d}_{5}$ are $4.19 \%$ for $\mathrm{m} / \mathrm{z} 104$ and $0.28 \%$ for $\mathrm{m} / \mathrm{z} 162$.

${ }^{\mathrm{b}}$ Average of triplicates.

"Normalized ion intensity data were used to calculate the CC data: the contribution of MDA- $\mathrm{d}_{5}$ to the intensities of ions designating MDA are $2.99 \%$ for $\mathrm{m} / \mathrm{z} 100$ and $0.068 \%$ for $\mathrm{m} / \mathrm{z} 158$; the contribution of MDA to the intensities of ions designating MDA- $\mathrm{d}_{5}$ are $8.09 \%$ for $\mathrm{m} / \mathrm{z} 104$ and $0.55 \%$ for $\mathrm{m} / \mathrm{z}$ 162.

measurement of ion intensities. For the purpose of this study, significant deviations resulting from the adoptation of ion-pair $\mathrm{m} / \mathrm{z} 100 / 104$ as the quantitation ion-pair can better illustrate the novel approach proposed for assessing the empirically determined CC data.

It should be noted that most analytical protocols would select the most favorable ion-pair to designate the analyte and the IS and adopt a multiple-point approach instead of the one-point calibration approach. One-point calibration is a two-point linear model using one calibrator and assuming that the response is " 0 " when the analyte's concentration is at $0 \mathrm{ng} / \mathrm{mL}$. This approach provides accurate quantitation data when the analyte's concentration in the test sample is at the vicinity of the concentration of the selected one-point calibrator. With only two data points available, onepoint calibration must adopt the linear model with a very limited linear range, especially when the CC of the selected ion-pair is significant. On the other hand, with multiple-point calibration, linear and other models, such as polynomial or hyperbolic, can be used to fit the observed data, thus compensating for CC effect that are highly significant at the lower and higher ends. This would results in a much wider calibration range [12].

\section{Effect of CC on Calibration Curve}

The ion intensity ratios shown in the second column of Table 3 are the empirically observed values for the ion-pairs designating MDA and MDA- $\mathrm{d}_{5}$. The concentrations shown in the third column are the empirically observed concentrations of these standards based on the ratios shown in the second column, using the 500 
$\mathrm{ng} / \mathrm{mL}$ standard as the calibration standard. The percentage figures shown inside parentheses in the third column are percentage deviations of the empirically observed concentrations from the true (or prepared) concentrations.

To best illustrate the effect of CC on quantitation result, one-point calibration approach was used to derive the observed concentration resulting from each ion intensity ratio. Calibration curve approach would have averaged out the positive/negative deviations exhibited by standards at the lower and higher ends of the curve, thus reducing the deviations that serve as the basis of this study. With one-point calibration, the observed concentration of a standard/specimen can deviate from its true value significantly when the CC of the adopted ion-pair is significant. Thus, for the standard with $4000 \mathrm{ng} / \mathrm{mL}$ analyte, $2017 \mathrm{ng} / \mathrm{mL}$ was observed adopting $\mathrm{m} / \mathrm{z} 100 / 104$ as the quantitation ionpair. The corresponding concentration was $3860 \mathrm{ng} / \mathrm{mL}$ when ion-pair $m / z$ 158/162 was adopted as the quantitation ion-pair.

As we have reported earlier [12], the CC phenomenon will cause the intensity ratio values shown in the second column to deviate from a linear relationship when plotted against their respective concentrations. This nonlinear relationship and need for correction has also been emphasized by Duncan et al. [8] as discussed in the Introduction section. Our objective for this part of the study is to evaluate the accuracy of the two sets of CC data that were derived from direct measurement without and with a normalization process. For this purpose, each set of the CC data was used to derive a set of ion intensity ratios for this series of standard solutions. The calculated intensity ratios were then used to derive the concentrations of this series of standards. With two sets of CC data, we have calculated two sets of intensity/concentration figures. These two sets of calculated ratio/concentration data are shown in the fourth/fifth and the sixth/seventh columns in Table 3.

Theoretically calculated data were derived with the following stipulations: (1) as justified in the second paragraph of this subsection, quantitation is based on one-point calibration, of which the concentrations of the analyte and the IS in the calibrator were both 500 $\mathrm{ng} / \mathrm{mL}$; (2) the intensities of the ions, designating the analyte and the IS, increase and decrease linearly with their concentrations; and (3) the CC values (i.e., analyte's contribution to the intensity of the ion designating the IS and the IS's contribution to the ion designating the analyte) as empirically determined, were applied. Stipulation " 2 " is true as shown by the data resulting from the $m / z$ 158/162 ion-pair. The most important aspect of this study is that if the empirically derived CCs were inaccurate, stipulation " 3 " would embed a systematic error in the calculated concentrations. This error would allow for assessing the trueness of the CC values as discussed in the next section.

With these stipulations in mind, a sample calculation (with $\mathrm{m} / \mathrm{z} 100$ for the analyte and $\mathrm{m} / \mathrm{z} 104$ for the IS) is shown below. At $500 \mathrm{ng} / \mathrm{mL}$, the average intensity ratio for $m / z 100\left(\mathrm{I}_{100}\right)$ to $104\left(\mathrm{I}_{104}\right)$ derived from the raw data for the 12 measurements (six for day 1 and six for day 2) shown in Table 2 was $I_{100} / I_{104}=0.5936 / 1$.

When the analyte's concentration is $4000 \mathrm{ng} / \mathrm{mL}$ and the IS's concentration remains at $500 \mathrm{ng} / \mathrm{mL}$, the $\mathrm{I}_{100} /$ $\mathrm{I}_{104}$ ratio without $\mathrm{CC}$ would have been $[0.5936 \times$ $(4000 / 500)] / 1$, or $4.749 / 1$ [instead of $(4000 / 500) / 1$, or $8 / 1$ ]. However, taking the $5.92 \%$ and $4.19 \%$ directlymeasured CC data into account, the resulting ion intensity ratio would have been

$\mathrm{I}_{100} / \mathrm{I}_{104}=(4.749+0.0592) /(1+4.749 \times 0.0419)=$ 4.0101. With this calculated ion intensity ratio, the resulting concentration of the analyte, $\mathrm{X}$, can be calculated as follows:

$0.5936 / 500=4.0101 / X ; X=4.0101 \times 500 / 0.5936=$ $3378 \mathrm{ng} / \mathrm{mL}$.

Thus, the calculated concentration of the analyte is (3378-4000)/4000, or $-15.6 \%$, lower than the expected value, $4000 \mathrm{ng} / \mathrm{mL}$. The theoretically calculated concentrations for the standards at other concentrations (and their deviations from the respectively expected values) were similarly calculated and placed in the fourth and fifth columns of Table 3.

Shown in the sixth and seventh columns of Table 3 are the same as the data shown in the fourth and the fifth columns, except that the ion intensity data, for ions $m / z 100$ and 104, and thereby derived normalized CC data, were used for calculation. Basically, the calculation is the same as shown above, with the exception that $\mathrm{I}_{100} / \mathrm{I}_{104}=1291998 / 1131499=1.1418$, while the CCs for the IS to the analyte and the analyte to the IS are $2.99 \%$ and $8.09 \%$, respectively.

\section{Graphic Presentation on the Evaluation of CC Data Based on the Consistency of the Empirically Observed and Theoretically Calculated Concentrations}

Shown in the second and the fourth columns of Table 3 are the empirically and the theoretically calculated concentration (using the $\mathrm{CC}$ data derived from raw ion intensity data). Deviations of these concentrations from the expected values (for standards at different concentration levels) were plotted in Figure 4(A). Clearly, the calculated concentrations (Figure 4(A)-(b) are not consistent with what have been actually observed, and their deviations from the expected values are even more significant than that derived from the empirically observed concentrations (Figure 4(A)-(a). This is a clear indication that the $\mathrm{CC}$ values derived from the raw ion intensity data and used as the basis for theoretical calculations to derive the concentrations shown in column 4 were inaccurate.

Could this deviation be caused by experimental errors, such as using different quantities of MDA and MDA- $\mathrm{d}_{5}$ or difference in the completion of the derivatization reactions for these two compounds? These 

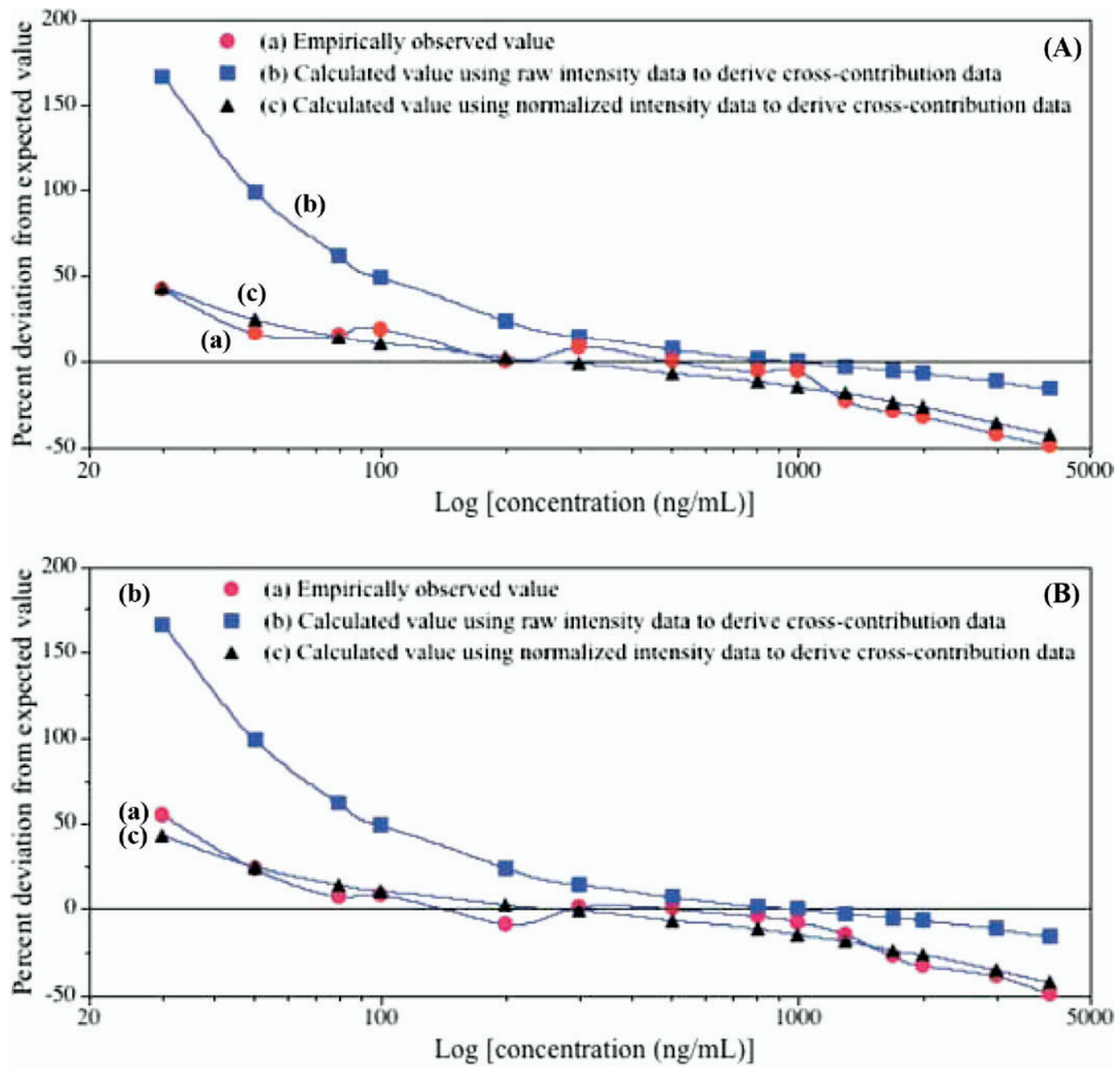

Figure 4. Comparison of errors derived from observed concentrations and those calculated with corrections of CC data derived from two methods, the MDA/MDA- $\mathrm{d}_{5}$ system ( $\mathrm{m} / \mathrm{z}$ 100/104). (A) and (B): results of the first and the second set of study; (a) deviation of empirically observed from the expected concentrations; (b) deviation of the theoretically calculated concentrations, using CC data $\left(5.92 \%\right.$ and $4.19 \%$ for the MDA/MDA- $\mathrm{d}_{5}$ system), derived from raw intensity data, from the expected concentrations; and (c) deviation of theoretically calculated concentrations, using CCs data $(2.99 \%$ and $8.09 \%$ for the MDA/MDA- $\mathrm{d}_{5}$ system), derived from normalized intensity data, from the expected concentrations.

possibilities were ruled out because the curve (Figure 4(A)-(c) generated by the deviation data shown in the seventh column of Table 3 exhibits excellent agreement with the curve derived from the empirically observed concentration data. Thus, CC data derived from the normalization process, but not from raw intensity data, can fully describe the observed MDA concentrations that were calculated based on experimentally observed ion intensity ratios. It is thus concluded that the ion intensity normalization process produce more accurate CC data.

To validate what have been stated above, another set of standards were prepared and experiments were performed. The resulting plots are shown in Figure 4(B). Figure 4(A) and (B) exhibit excellent agreement.

To further prove the normalized intensity data can be reliably used for the calculation of CC values in other analyte/IS systems, the TMS-derivatized HM/ HM- $\mathrm{d}_{6}$ system (see Figure 1B for full-scan mass spectra and molecular information), with an ion-pair exhibiting significant CC, was randomly selected as another example. Studies parallel to that adopted for the MDA/MDA- $\mathrm{d}_{5}$ system were performed. Adopting $\mathrm{m} / \mathrm{z} 234 / 240$ (Figure 3) as the quantitation ionpair designating $\mathrm{HM} / \mathrm{HM}-\mathrm{d}_{6}$, the CC of the IS to the analyte and the analyte to the IS, which were calculated based on the raw ion intensity data, were 1.14 and 9.83, respectively. The corresponding CC data using the normalized data were 2.70 and 4.15 , respectively. The resulting curves are shown in Figure 5. It is interesting to note that curve (b) starts higher than curves (a) and (c) in Figure 4A and B, while the reverse is true in Figure 5, reflect that the normalization processes in the MDA/MDA- $\mathrm{d}_{5}$ and the HM/ 


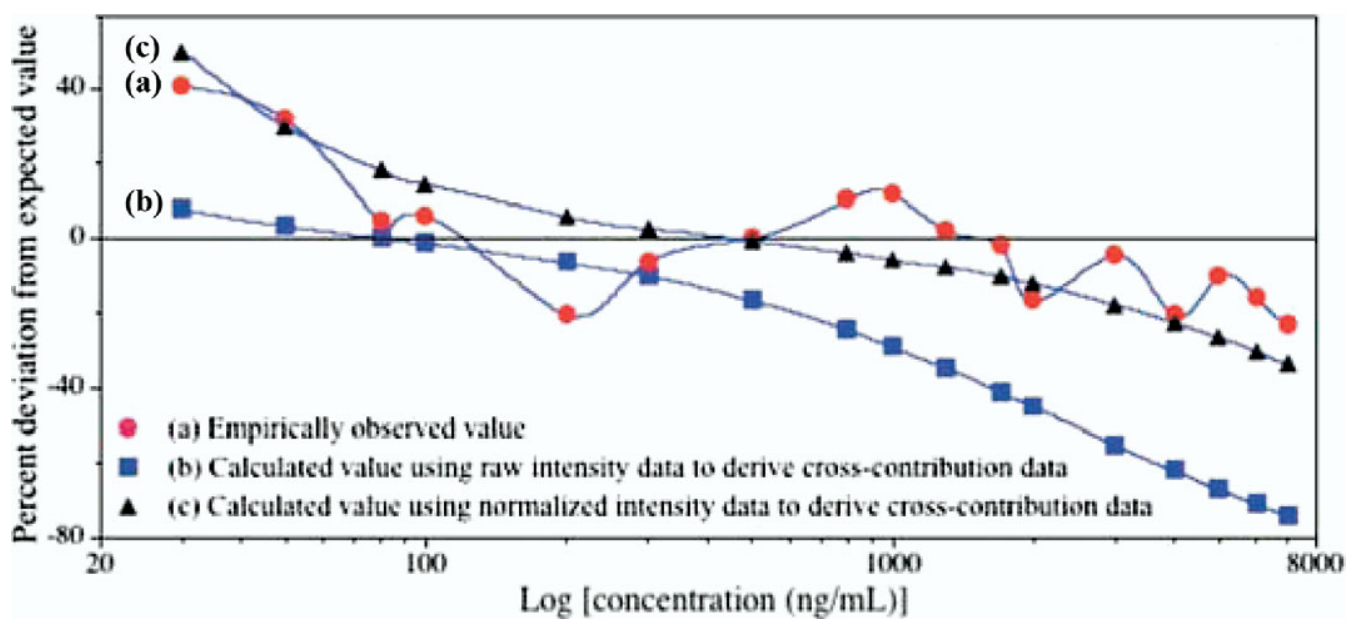

Figure 5. A parallel set of data derived from the study on the HM/HM-d $\mathrm{d}_{6}$ system $(\mathrm{m} / z 2234 / 240)$; (a) deviation of empirically observed from the expected concentrations; (b) deviation of the theoretically calculated concentrations, using CC data $(1.14 \%$ and $9.83 \%$ for the hydromorphone/hydromorphone- $\mathrm{d}_{6}$ system), derived from raw intensity data, from the expected concentrations; and (c) deviation of theoretically calculated concentrations, using CCs data $(2.70 \%$ and $4.15 \%$ for the hydromorphone/hydromorphone- $\mathrm{d}_{6}$ system), derived from normalized intensity data, from the expected concentrations.

$\mathrm{HM}-\mathrm{d}_{6}$ systems adjusted the relative analyte/IS ion intensity in opposite directions.

It is noted that curves (a) and (c) in Figure 5 do not coincide as well as the corresponding curves in Figure 4. We believe this does not invalidate the application of the normalization approach to the $\mathrm{HM} / \mathrm{HM}-\mathrm{d}_{6}$ system; instead, it is a reflection of more significant experimental errors incurred in the $\mathrm{HM} / \mathrm{HM}-\mathrm{d}_{6}$ study. Further study will be conducted in the future to include other analyte/IS systems and CC data derived from other methods [10].

In conclusion, the ion intensity normalization process has been proven effective for generating accurate $\mathrm{CC}$ data, at least for the MDA/MDA- $\mathrm{d}_{5}$ system, and perhaps more importantly, the CC evaluation approach developed in this study is proven effective. This result further indicates that potential H/D isotope effect in the ion fragmentation process does not play an important role in the generation of ions adopted in our study. This is not surprising as the ion-pairs adopted for quantitation purpose were generated without the breaking of $\mathrm{C}-\mathrm{H} / \mathrm{C}-\mathrm{D}$ bonds in the corresponding analyte/IS molecular framework.

\section{Effects of CC on Achievable Linear Range}

In theory, if the intensity of the ion designating the analyte includes contribution by the IS, the percentage of the intensity of this ion derived from the IS becomes increasingly significant as the analyte's concentration is lowered. Consequently, the achievable limit of quantitation would be at a higher level when the contribution of the IS to the intensity of the ion designating the analyte becomes larger. Similarly, if the analyte makes a significant contribution to the intensity of the ion des- ignating the IS; then, as the analyte's concentration becomes higher, the observed concentration will become increasingly lower than the true value.

Shown in Table 3 are data related to the effect of CC on achievable linear range. Two sets of ion-pairs (one with significant $C C$, one with negligible $C C$ ) from the MDA/MDA- $\mathrm{d}_{5}$ system were adopted for illustration. For the $m / z$ 100/104 ion-pair (upper section of the table), based on the raw ion intensity data, the CC of the IS to the analyte and the analyte to the IS were $5.92 \%$ and $4.19 \%$, respectively. With these levels of CC, the acceptable concentration range (with less than $20 \%$ deviations from the expected values) derived from the empirically observed data (columns 1 and 2 in Table 3 ) is limited to 200-1000 $\mathrm{ng} / \mathrm{mL}$, using $500 \mathrm{ng} / \mathrm{mL}$ as the one-point calibration standard.

The lower section of Table 3 provides the parallel quantification data adopting an ion-pair $(\mathrm{m} / \mathrm{z}$ 158/ 162) with much lower CC. Based on raw ion intensity data, the CC of the IS to the analyte and the analyte to the IS were $0.14 \%$ and $0.28 \%$, respectively. With these levels of CC data, the deviations of the empirically observed concentrations within the entire range studied $(30-4000 \mathrm{ng} / \mathrm{mL}$ ) were all lower than $20 \%$ (column 2 in Table 3).

In conclusion, this study has developed a novel approach to assess the accuracy of the CC data between the ions designating the analyte and the deuterated IS, and concluded that the normalized ion intensity data can be reliably used for the calculation of the CC values, at least for the systems studied. Empirically and theoretically calculated data both indicate that an ion-pair with $\sim 5 \%$ (or higher) CC will result in a very limited linear calibration range. 


\section{Acknowledgments}

The authors gratefully acknowledge financial support from (Taiwanese) National Bureau of Controlled Drugs (DOH-96NNB-1004) and National Science Council (NSC 95-2745-M-242003-URD), and technical support provided by Ms. Meng-Yen $\mathrm{Wu}$.

\section{References}

1. U.S. Department of Health and Human Services. Mandatory Guidelines for Federal Workplace Drug Testing Programs. Fed. Reg. 1988, 53, 11970-11989.

2. Holland, J. F.; Sweeley, C. C.; Thrush, R. E.; Teets, R. E.; Bieber, M. A. Anal. Chem. 1973, 45, 308-314.

3. Garland, W. A.; Barbalas M. P. Applications to Analytical Chemistry: An Evaluation of Stable Isotopes in Mass Spectral Drug Assays. J. Clin. Pharmacol. 1986, 26, 412-418.

4. Pickup, J. F.; McPherson, K. Theoretical Considerations in Stable Isotope Dilution Mass Spectrometry for Organic Analysis. Anal. Chem. 1976, 48, 1885-1890.

5. Thorne, G. C.; Gaskell, S. J.; Payne, P. A. Approaches to the Improvement of Quantitative Precision in Selected Ion Monitoring: High Resolution Applications. Biomed. Mass Spectrom. 1984, 11, 415-420.

6. Bush, E. D.; Trager, W. F. Analysis of Linear Approaches to Quantitative Stable Isotope Methodology in Mass Spectrometry. Biomed. Mass Spectrom. 1981, 8, 211-218.
7. Barbalas, M. P.; Garland, W. A. A Computer Program for the Deconvolution of Mass Spectral Peak Abundance Data from Experiments Using Stable Isotopes. J. Pharm. Sci. 1991, 80, 922-927.

8. Duncan, M. W.; Gale, P. J.; Yergey, A. L. The Principles of Quantitative Mass Spectrometry; Rockpool Productions: Denver, CO 2006; p 97.

9. Liu, R. H.; Foster, G. F.; Cone, E. J.; Kuma, S. D. Selecting an Appropriate Isotopic Internal Standard for Gas Chromatography/Mass Spectrometry Analysis of Drugs of Abuse-Pentobarbital Example. J. Forensic Sci. 1995, 40, 983-989.

10. Chang, W.-T.; Lin, D.-L.; Liu, R. H. Isotopic Analogs as Internal Standards for Quantitative Analyses by GC/MS-Evaluation of CrossContribution to Ions Designated for the Analyte and the Isotopic Internal Standard. Forensic Sci. Int. 2001, 121, 174-182.

11. Liu, R. H.; Lin, T.-L.; Chang, W.-T.; Liu, C.; Tsay, W.-I.; Li, J.-H.; Kuo T.-L. Isotopically Labeled Analogues for Drug Quantitation. Anal. Chem. 2002, 74, 618A-626A

12. Whiting, T.C.; Liu, R. H.; Chang, W.-T.; Bodapati, M. R. Isotopic Analogs as Internal Standards for Quantitative Analyses of Drugs/ Metabolites by GC/MS-Nonlinear Calibration Approaches. J. Anal. Toxicol. 2001, 25, 179-189.

13. Wang, S.-M.; Chye, S.-M.; Liu, R. H.; Lewis. R. J.; Canfield, D. V Roberts J. Mass Spectrometric Data of Commonly Abused Amphetamines and Their Derivatives-Cross Contributions of Ion Intensity Between the Analytes and Their Isotopically Labeled Analogs. Forensic Sci. Rev. 2005, 17, 67-166.

14. Liu, R. H.; Baugh, L. D.; Allen, E. E.; Salud, S. C.; Fentress, J. C.; Chadha, H.; Walia, A. S. Isotopic Analogue as the Internal Standard for Quantitative Determination of Benzoylecgonine: Concerns with Isotopic Purity and Concentration Level. J. Forensic Sci. 1989, 34, 986-990. 